\title{
Spatial heterogeneity of soil properties and solute transport characteristics and their correlations in degraded wetland soils
}

\author{
Yinghu Zhang ${ }^{1}$, Jinchi Zhang ${ }^{1}$, Zhenming Zhang², and Mingxiang Zhang² \\ ${ }^{1}$ Nanjing Forestry University \\ ${ }^{2}$ Beijing Forestry University
}

June 23, 2021

\begin{abstract}
Soil properties have a significant influence on solutes redistribution in the soil vadose zones. The aim of this study was to assess the relevance of soil properties for solute transport characteristics in degraded wetland soils using 72 undisturbed soil columns from two experimental fields located in Robinia pseudoacacia (CH) and Tamarix chinensis (CL) communities. Combining soil column tracer experiments, all experiments were conducted under the same initial and boundary conditions using Brilliant Blue FCF as a conservative tracer. Solute transport characteristics were described by four measures of dye solution steady infiltration rate of effluents, dye solution concentration of effluents, soil column dye staining patterns, and cumulative dye solution leaching. Numerical modeling by the dual-permeability model in HYDRUS-1D was used to simulate the proportion of cumulative dye solution leaching from soil macropore flow. This study showed that basic soil properties exhibited a significant difference at $\mathrm{CH}$ site and at CL site. Dye solution steady infiltration rate of effluents at $\mathrm{CH}$ site decreased with soil depth, but increased at first and then decreased with soil depth at CL site. Dye solution concentration of effluents both at CH site and at CL site decreased nonlinearly with soil depth. Soil column dye staining patterns were significantly different among different soil locations, indicating the largest dark blue staining domains from soil depth of 0-10 cm at CH site and 20-40 cm at CL site. The proportion of cumulative dye solution leaching from soil macropore flow was from 37.6 to $61.1 \%$ at $\mathrm{CH}$ site, whereas from 0 to $99.9 \%$ at CL site. Basic soil properties played inconsistent roles in solute transport characteristics. The understanding of soil properties and its correlation with solute transport characteristics is the first step for degraded wetland restoration and development. Some alternative solutions of wetland restoration are proposed for managers.
\end{abstract}

\section{Hosted file}

manuscript.docx available at https://authorea.com/users/421305/articles/527382-spatialheterogeneity-of-soil-properties-and-solute-transport-characteristics-and-their-

correlations-in-degraded-wetland-soils 


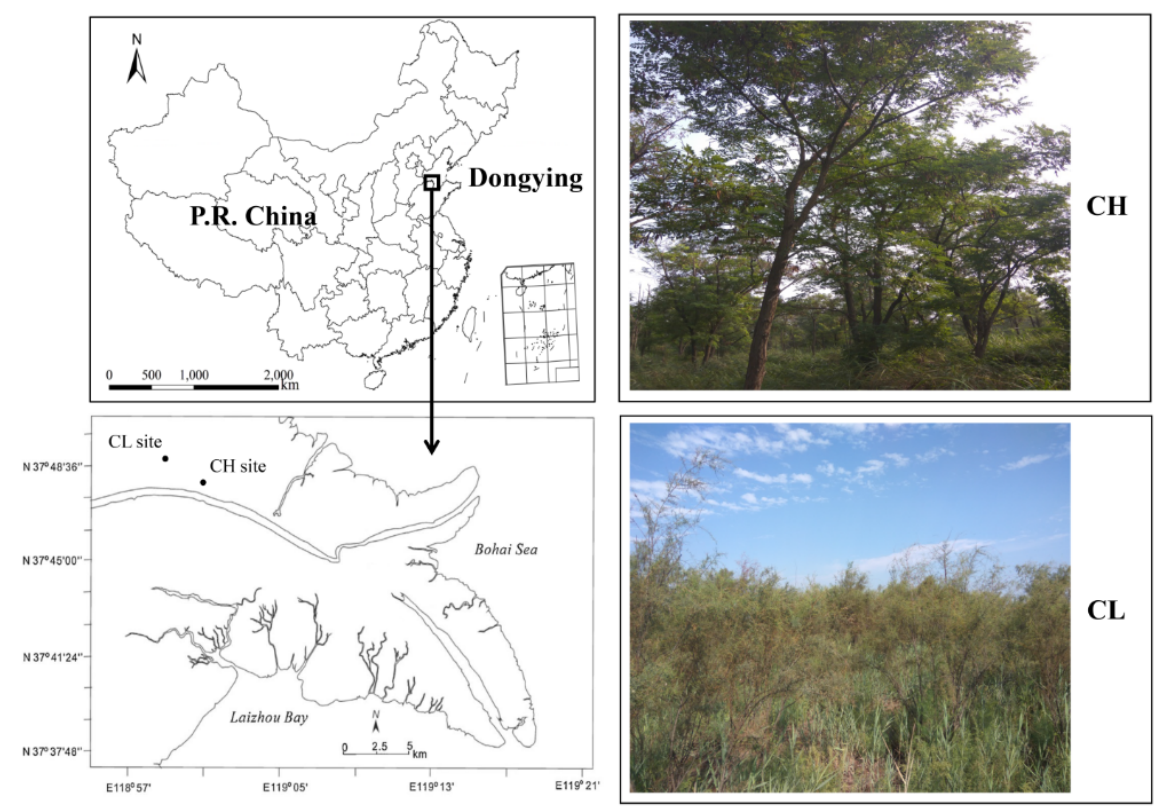



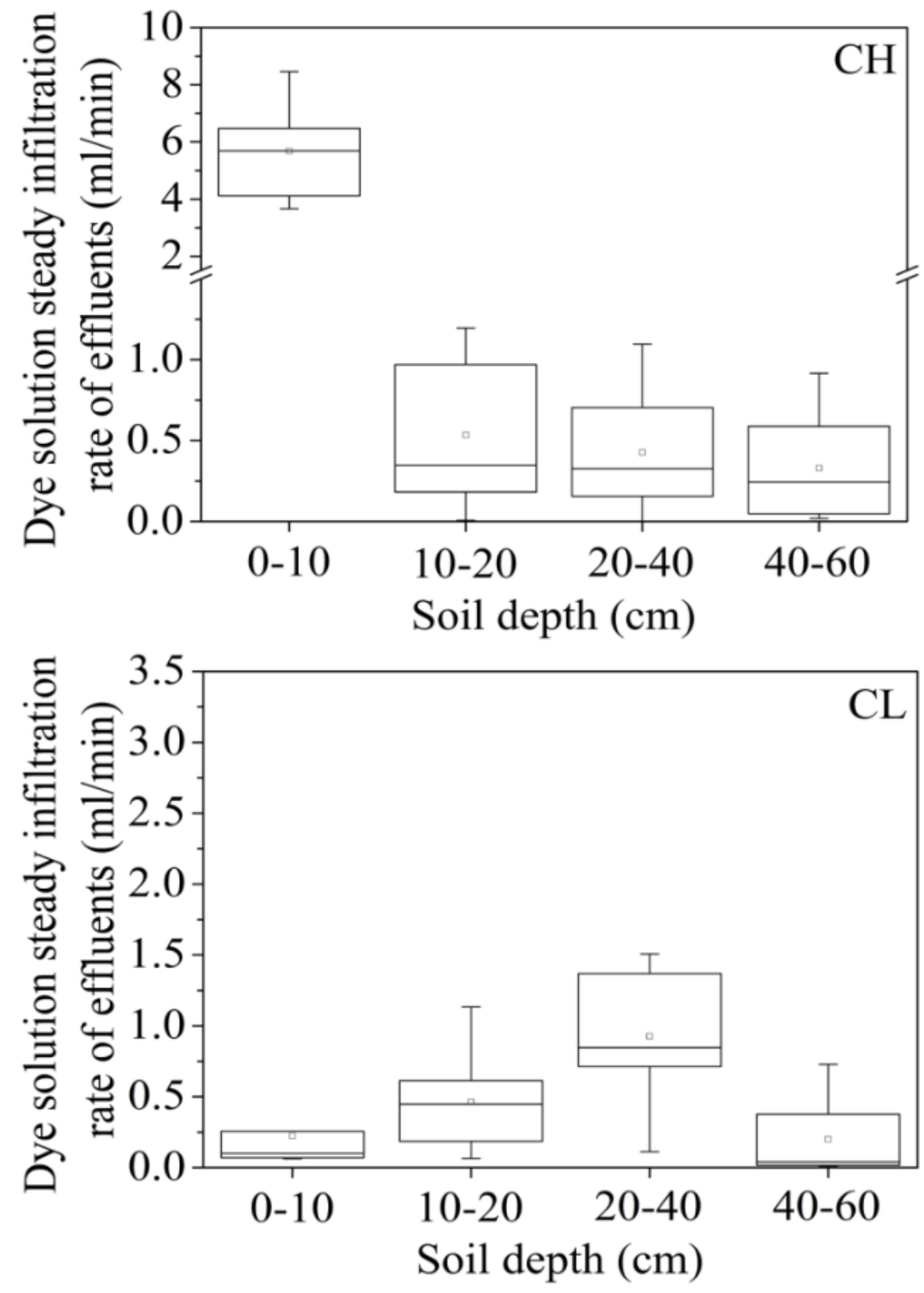

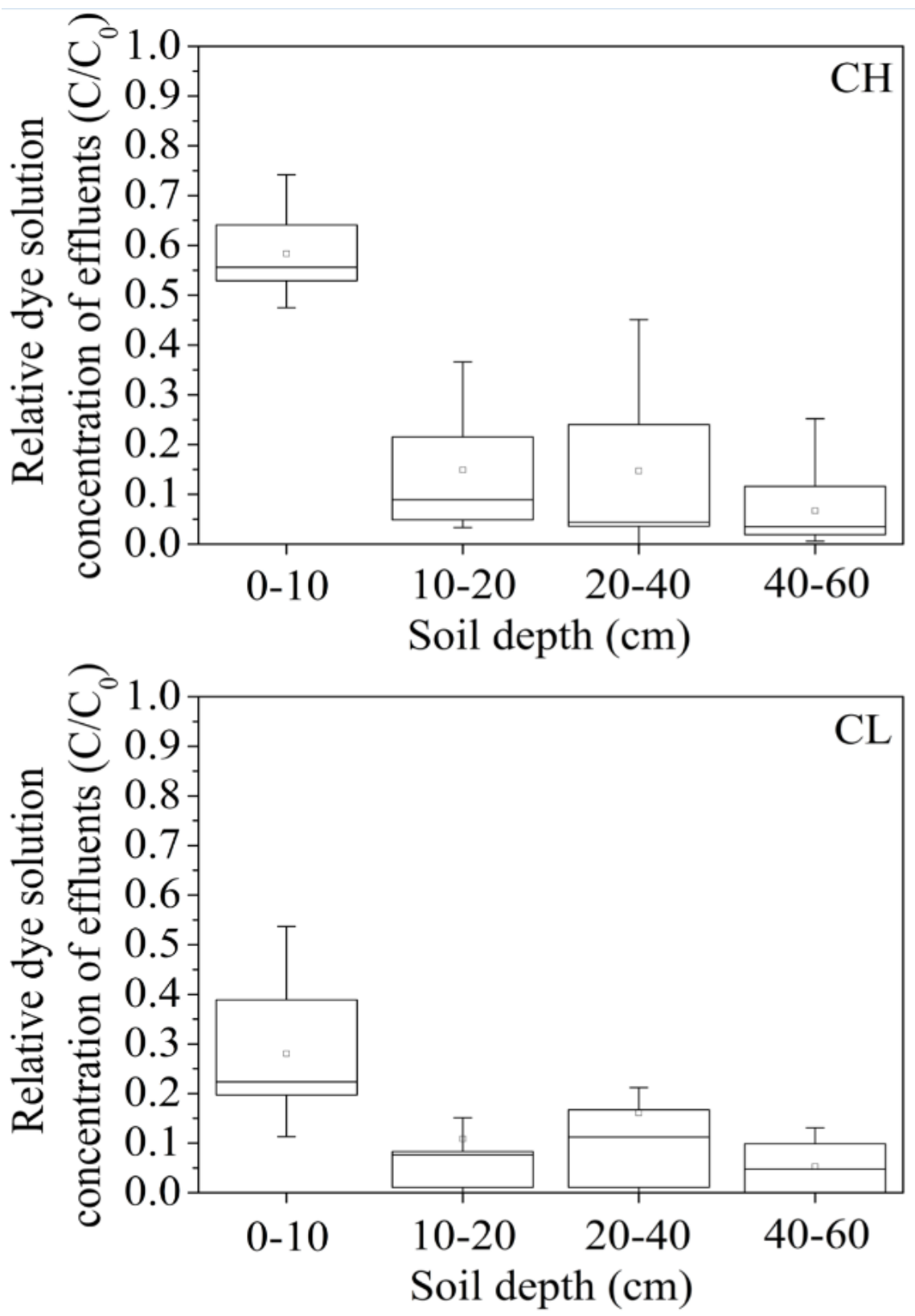

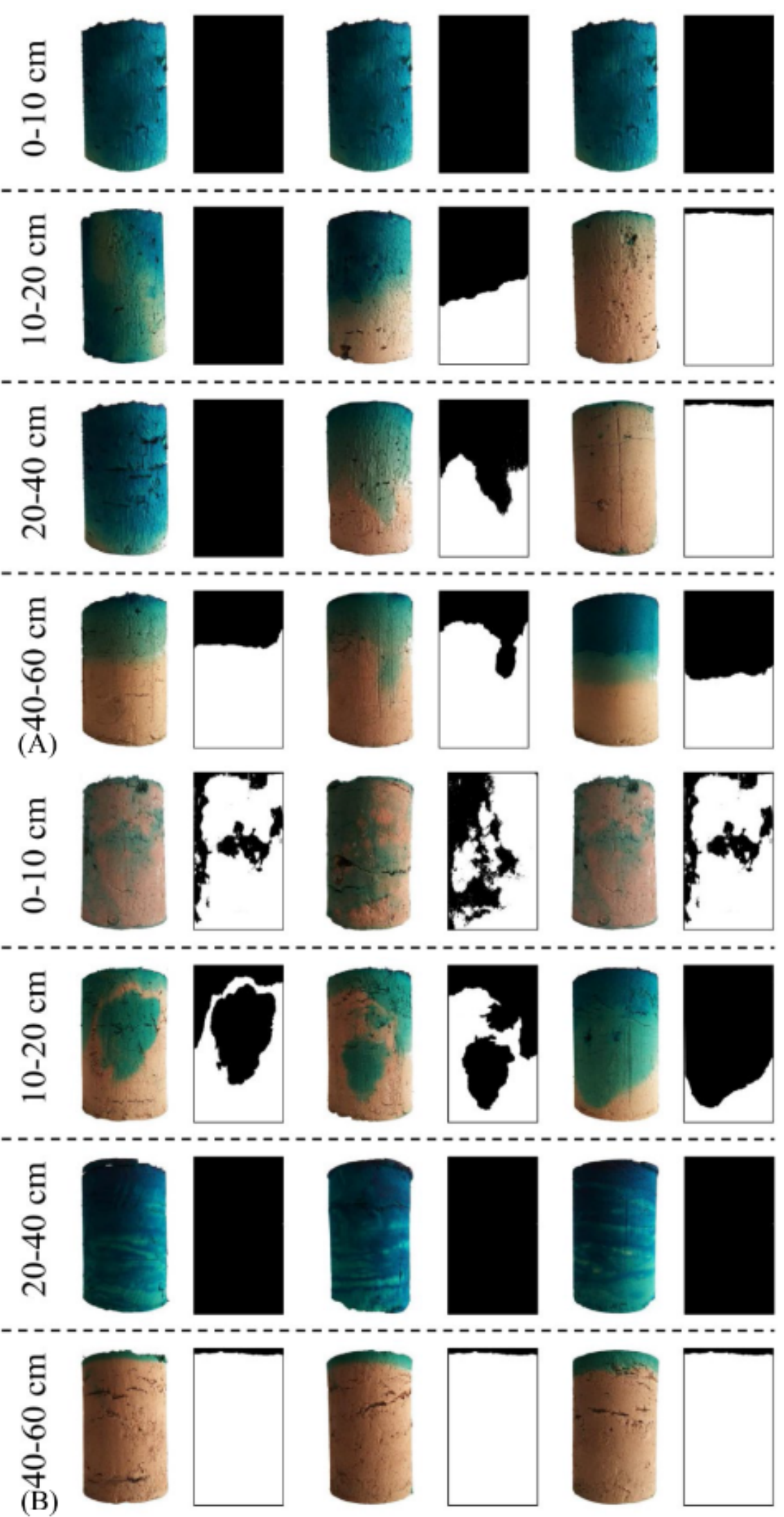

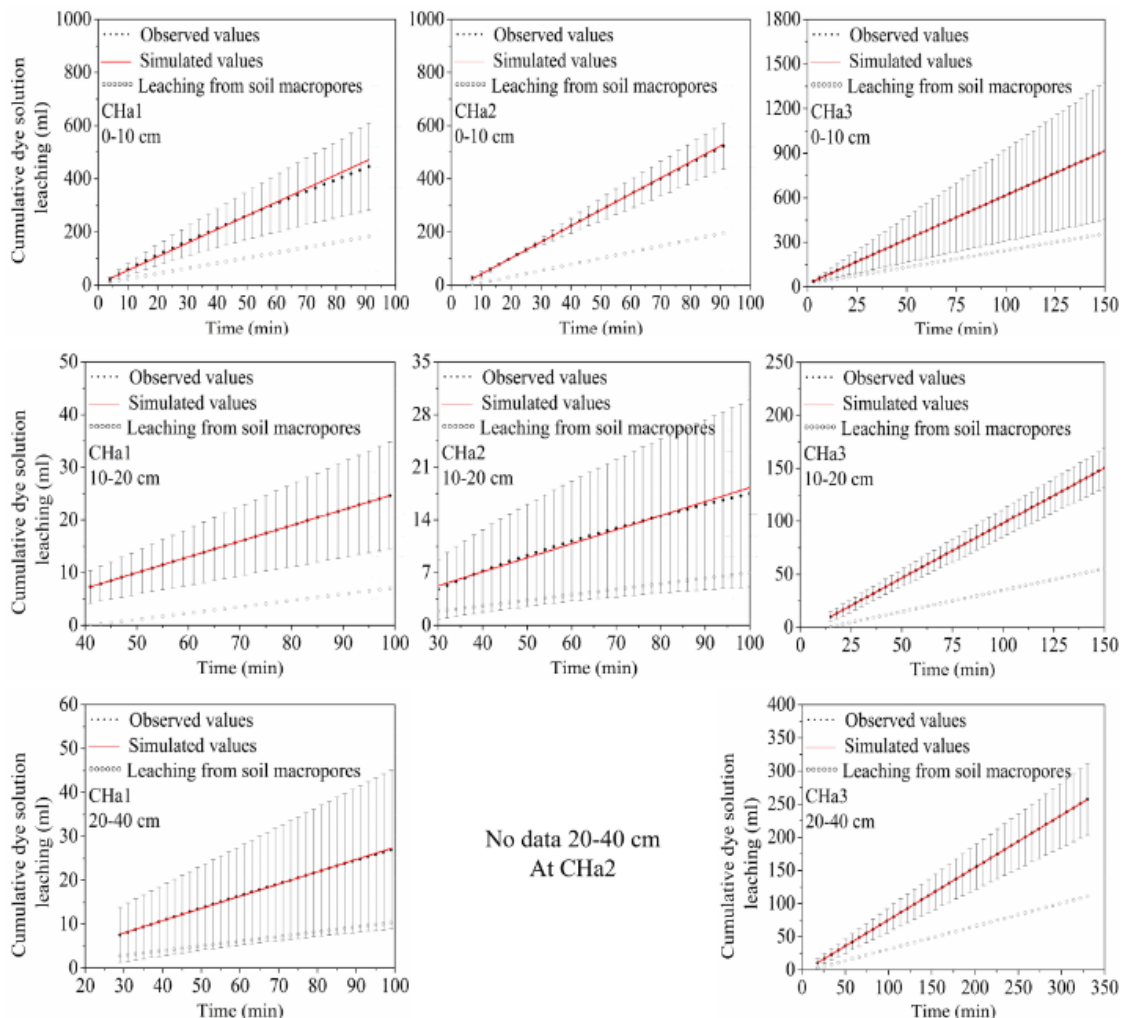

Time (min)
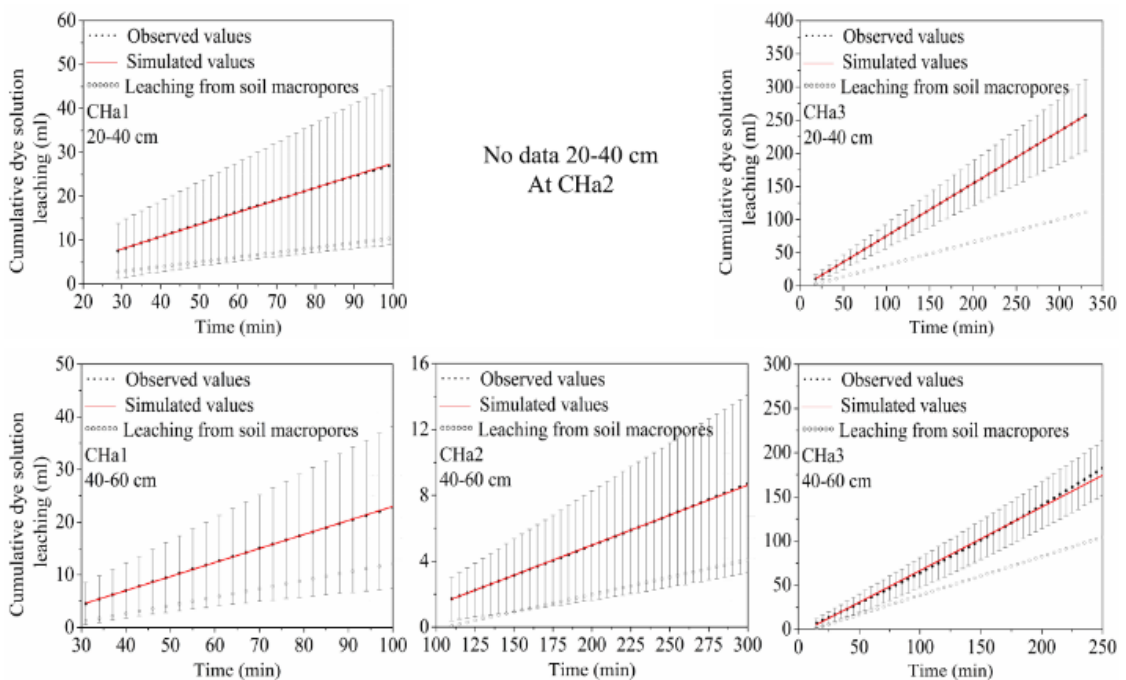

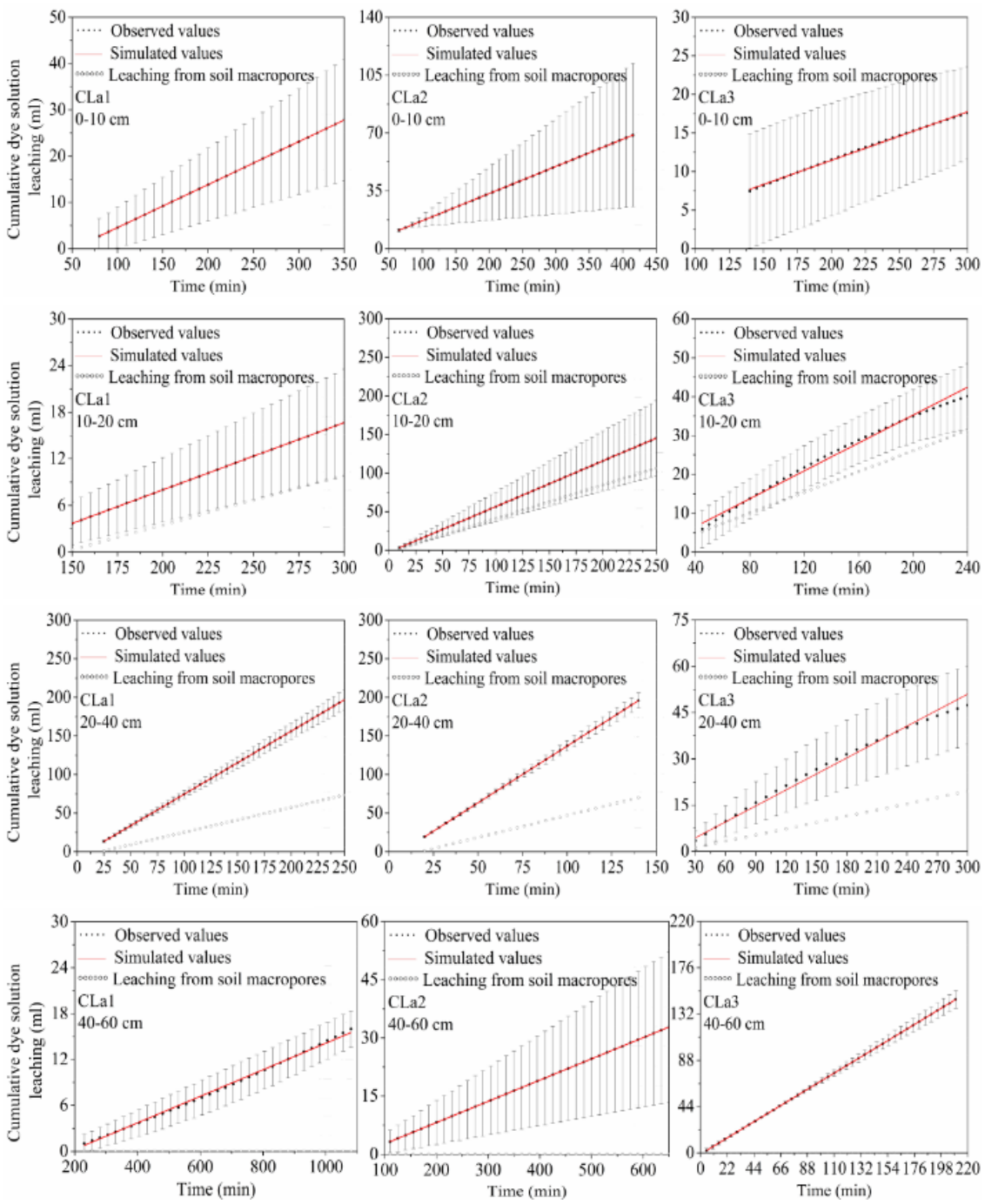